\title{
Temporäre lokale Ökonomien als Ausdruck des gesellschaftlichen Wandels. Eine synoptische Übersicht
}

\section{Temporary local economies as an expression of social change. A synoptic overview}

https://doi.org/10.2478/rara-2020-0028

Eingegangen: 5. März 2020, Angenommen: 23. September 2020

Kurzfassung: Mit dem Begriff „temporäre lokale Ökonomien“ lässt sich die Gesamtheit aller auf die Entwicklung eines Quartieres und einer Region bezogenen wirtschaftlichen Aktivitäten in einem temporären Arrangement zusammenfassen. Diese kennzeichnet die spannungsreiche Kombination von ökonomischem Handeln und Altagsleben in einem stadtregionalen Kontext. Ziel dieses Beitrags ist es, die temporären lokalen Ökonomien in ihren fachlichen Diskursen überblicksartig darzustellen und anhand einer beispielhaften Kurzdarstellung (Bikini Berlin) Einblicke in stadtregionale Entwicklungslinien zu geben.

Schlüsselwörter: Temporäre lokale Ökonomien, stadtregionale Entwicklung, Pop-up-Stores, Berlin

Abstract: The term "temporary local economies" can be used to summarise all of the economic activities related to the development of a location and a region in a temporary arrangement. This characterises the exciting combination of economic activity and everyday life in an urban and regional context. The aim of this paper is to provide an overview of temporary local economies in their academic perspectives and to give insights into urban regional development lines using an exemplary brief case study (Bikini Berlin).

Keywords: Temporary local economies, Urban and regional development, Pop-up stores, Berlin

\footnotetext{
*Corresponding author: Dr. Petra Lütke, Westfälische Wilhelms-Universität Münster, Institut für Geographie, Heisenbergstraße 2,
} 48149 Münster, Deutschland, E-Mail: petra.luetke@uni-muenster.de 


\section{Einleitung: Lokale Ökonomien}

Der Begriff der „lokalen Ökonomien“ ist mittlerweile sowohl in unterschiedlichen akademischen Diskursen als auch in kommunalpolitischen und stadtplanerischen Initiativen nicht mehr wegzudenken. Obwohl kein einheitliches Verständnis von lokalen Ökonomien existiert, lassen sich verschiedene Aspekte zusammenfassen, die allgemein Anerkennung finden. Ältere Definitionen umschreiben lokale Ökonomien bisher im weiteren Sinne als die "Gesamtheit aller auf die Entwicklung eines Ortes (einer Region) bezogenen wirtschaftlichen Aktivitäten national bzw. international" (Birkhölzer 2000: 58). Der ursprünglich aus Großbritannien stammende Begriff fand seinen Einzug in die wissenschaftliche Debatte in Deutschland Mitte der 1980er-Jahre. Dabei handelte es sich um einen Ansatz, der im sozialpolitischen Kontext des Strukturwandels formelle und informelle sowie produktive und reproduktive wirtschaftliche Aktivitäten umfasste. Dieser integrative Ansatz und das stark an der Praxis ausgerichtete Begriffsverständnis finden sich auch heute noch in verschiedenen interdisziplinären Forschungsfeldern zu Städten und Regionen wieder, die "insbesondere die Trennung in ,rein“ wirtschaftliche, soziale und ökologische Sichtweisen aufzuheben versuch[en]“ (Birkhölzer 2000: 58). Güleş und Schultheis (2019: 14) kritisieren diese holistische Perspektive auf wirtschaftliche Aktivität eines Ortes, da so keine Differenzierung zwischen den unterschiedlichen Konzepten lokaler Ökonomien möglich sei, die diese aber gerade ausmachten, wie beispielsweise soziale Ökonomien, solidarische Ökonomien, Dritter Sektor, Gemeinwesenökonomien oder ethnische Ökonomien. Die diesem Beitrag zugrunde liegende Definition von lokalen Ökonomien orientiert sich stärker an den Diskursen des Social Entrepreneurhip, die "gesellschaftliche, also soziale oder ökologische, Probleme mit unternehmerischen Mitteln lösen. Social Entrepreneurship (Soziales Unternehmertum) ist dabei auf gesellschaftliche Wertschöpfung ausgerichtet und nicht auf die Maximierung finanzieller Rendite" (Rummel 2011: 15). Dieser stärker sozialwissenschaftlich ausgerichtete Begriff lokaler Ökonomien ist weiterhin gekennzeichnet durch wirtschaftliches Handeln, das sowohl auf unternehmerische Prinzipien rekurriert, leistungsbasierte Einkommen erzielt und zugleich im Quartier sozial verankert ist (Henn/Behling 2020: 7).

In diesen Interpretationen wurden und werden lokale Ökonomien in Wissenschaft und Planungspraxis nahezu ausschließlich als permanente Einheiten konzeptualisiert, obwohl gerade auch temporäre Arrangements wirtschaftlicher Aktivität in stadtregionalen Kontexten an Bedeutung gewonnen haben. So kommt den weitgehend ortsgebundenen temporären lokalen Ökonomien vor allem bei der Stabilisierung benachteiligter Quartiere eine wichtige Funktion zu, indem etwa durch Zwischennutzungen leerstehender Gebäude oder Flächen kurzfristig neue ökonomische Nutzungen entstehen.

Ziel dieses Beitrags ist es, die temporären lokalen Ökonomien in ihren fachlichen Diskursen überblicksartig darzustellen und auf dieser Grundlage die jeweilige gesellschaftsstrukturelle raumzeitliche Veränderung zu analysieren. Hierdurch soll ein vertieftes Verständnis von bisher wenig untersuchten ephemeren Arbeits- und Freizeiträumen auf der Quartiersebene erzielt werden. Der Beitrag ist wie folgt strukturiert: In Kapitel 2 werden die konzeptionell-theoretischen Zugänge zum Phänomen temporärer lokaler Ökonomien erläutert. Unterschieden wird zwischen wirtschaftlicher Betätigung und Temporalität einerseits und gesellschaftsstrukturellen raumzeitlichen Veränderungen andererseits. In Kapitel 3 wird der Forschungsstand zu temporären lokalen Ökonomien und deren beobachtbaren Phänomenen diskutiert und in einer synoptischen Übersicht kondensiert. Die darauf folgende Darstellung eines Fallbeispiels in Kapitel 4 greift exemplarisch einen stadtregionalen Diskurs temporärer lokaler Ökonomien auf. In Kapitel 5 werden die Ergebnisse zusammengefasst und abschließend weitere Forschungsdesiderate aufgezeigt.

\section{Konzeptionelle Zugänge zu temporären lokalen Ökonomien}

\subsection{Wirtschaftliche Aktivität und Temporalität}

Ein verbindender Blick auf wirtschaftliche Aktivität und Temporalität ist in unterschiedlichen (geographisch-)theoretischen Konzeptionen zu finden. Sieht man von den klassischen Ansätzen der Time Geography der schwedischen Lund-Schule (Hägerstrand 1970; Hägerstrand 1975; Lenntorp 1999), der Aktionsraumforschung (z. B. Scheiner 1998) und einigen weiteren Ansätzen (z. B. May/Thrift 2001; Kramer 2005; Läpple/Mückenberger/Oßenbrügge 2010) ab, sind eher indirekte Verweise auf das Thema ökonomisches Handeln und Temporalität auszumachen. So sind etwa relationale (Massey 1984; Massey 2005; Bathelt/Glückler 2011) oder performative Ansätze (Kaiser/ Nikiforova 2008) zu nennen, die Temporalität in ihre ökonomischen Überlegungen mit einbeziehen (Kymäläinen/ 
Nordström 2010). Bestimmte temporäre Orte oder Events zeigen sich dabei auch als zentrale organisierende Einheiten ökonomischer Prozesse (Florida 2002; Bathelt/ Glückler 2011). Ibert und Thiel (2009) regen daher an, die zeitliche und die räumliche Dimension systematischer als bisher miteinander zu verschränken. Ausgangspunkt ihrer Analysen sind wissensbasierte Ökonomien, in denen Wissen als wichtige Ressource sozioökonomischer Maßnahmen fungiert. Dabei geht es weniger darum, räumlich unterschiedliche Bedingungen der Wissensproduktion zu identifizieren, als vielmehr um das Verstehen des Prozesses in seiner Raum-Zeitlichkeit (Oinas/Malecki 2002; Törnqvist 2004). In der Organisations- und Managementforschung wird in einer eigenständigen Perspektive von Temporalität und Organisation ein Defizit gesehen, welches in Forschung und Praxis noch nicht ausreichend berücksichtigt wurde (Koch/Sydow 2013: VII; Elbe/ Peters 2016: 2). In der Projekt- und Netzwerkforschung wird Temporalität oftmals als Prozess thematisiert ( $z$. B. Padgett/Powell 2012).

Eine Vielzahl an Äußerungen betonen das Verschwimmen von Zeit und Raum in seiner herkömmlichen Wahrnehmung, wie etwa die "time-space compression“ (Harvey 1990), die „Entnationalisierung von Raum und Zeit" (Sassen 2002) oder sagen gar das „Ende der Temporalität" (Jameson 2003) vorher. Diese parallellaufenden Zeitverhältnisse reflektieren den Zusammenbruch der Zeitlichkeiten in den sogenannten großen Erzählungen (Rilling 2014: 35) und identifizieren raumzeitliche Entkopplungen, in denen alles jederzeit und überall verfügbar sein soll. Castells (2002) hob die fundamentale Transformation von Raum und Zeit durch das Aufkommen des "multimedialen Kommunikationssystems“ hervor, indem er betonte: „Örtlichkeiten werden entkörperlicht und verlieren ihre kulturelle, historische und geografische Bedeutung. [...] Dadurch entsteht ein Raum der Ströme anstelle eines Raums der Orte. Die Zeit wird in dem neuen Kommunikationssystem ausradiert, wenn Vergangenheit, Gegenwart und Zukunft programmiert werden können, um miteinander in ein und derselben Botschaft zu interagieren. Der Raum der Ströme und die zeitlose Zeit sind die materiellen Grundlagen einer neuen Kultur, welche die Verschiedenheit der historisch überkommenen Systeme der Repräsentation überschreitet und in sich einschließt: die Kultur der realen Virtualität, wo Glaubenmachen Glauben an das Machen ist" (Castells 2002: 429, Hervorhebung im Original). Neben der sehr treffenden Darstellung des Einflusses des multimedialen Kommunikationssystems auf die Kategorien Zeit und Raum zeigt Castells „Raum der Ströme“ (1996) aber auch auf, dass es eine andere Wahrnehmung bzw. einen anderen Aggregatzustand von Zeit gebe, der stark an Baumans „Flüchtige Moderne“ (2007) erinnert. Diese andere Lesart gesellschaftlicher Veränderungen der Spätmoderne ${ }^{1}$, wie etwa die wachsende Dynamik, steigende Komplexität oder fluide Identitäten, lässt Zeit eben nicht verschwinden, sondern als unterschiedliche Qualitäten zwischen den Aggregatzuständen balancieren.

Liest man diese spätmodernen Entwicklungen in einem lokalen Kontext, so ist beispielsweise eine zunehmend wachsende Konsumorientierung der innerstädtischen Räume in Großstädten zu beobachten. Bedingt durch den Bevölkerungsanstieg in den Vorstädten, stellen etwa die kurzen Aufenthalte in den Innenstädten für viele Menschen ein Konsumabenteuer in spektakulär inszenierten flüchtigen urbanen Räumen dar (Wood 2003: 71). Auch in Bezug auf lokale Ökonomien sind vornehmlich durch das Ephemere neue Formen der Umgestaltung und der reflexiven Aneignung stadtregionaler Räume zu beobachten. Wiederkehrende soziale Praktiken führen zu gesellschaftlichen Dynamiken, die soziale Strukturen verändern und damit Gesellschaft insgesamt transformieren (Giddens 1992: 53). In einer Zeit, in der der Coffee to go ein Synonym für den urbanen Lebensstil geworden ist, scheinen sich die Beziehungsmuster zwischen Arbeits- und Freizeiträumen im städtischen Kontext stärker denn je zu verändern. Die Symbiose aus vorübergehendem Kaffeegenuss und vermeintlicher Geschäftigkeit avanciert zur symbolischen Darstellung eines individualisierten Lebensstils im öffentlichen Raum (Klamt 2012: 775). Diese Alltagssituation verdeutlicht einerseits eindrücklich die kontroversen Diskurse um die Neoliberalisierung der Stadt, wie etwa die Privatisierung und die Ökonomisierung öffentlicher Räume in der spätmodernen Stadt. Sie zeigt aber andererseits, dass eine eindeutige Trennung von Arbeits- und Freizeitwelt sowie privatem und öffentlichem Raum nicht mehr möglich ist und flüchtige ephemere Arbeits- und Freizeiträume entstehen. Das Bourdieu'sche Soziale und Ökonomische Kapital (1992) verschmilzt temporär zu einer "Ökonomie der Aufmerksamkeit" (Franck 1998). Gleichzeitig zeigt die Hybridisierung öffentlicher und halb-

1 Bei der Begriffsverwendung ist zwischen Postmoderne und Spätmoderne zu unterscheiden: Der Einsatz des Begriffs Postmoderne wird nach Rosa (2017) stärker aus einer kulturellen Perspektive verwendet, deren Diskurse überwiegend kulturwissenschaftlich-philosophisch geprägt sind. Dieser Begriff legt stärker das Eintreten eines qualitativ Neuen nahe, während der Begriff der Spätmoderne eher eine neue Gestalt des Alten (eben der Moderne) nahelegt und eine strukturelle Perspektive impliziert, die Kontexte der Analyse struktureller Veränderungen der modernen Gesellschaft in den Blick nimmt (Rosa 2017: 49 f.). 
öffentlicher Räume, dass sie zu einem konstitutiven Teil des ökonomischen Handelns werden (Ibert/Thiel 2009: 218). Nach Florida (2002) verbindet sich der erste Ort (privater Raum) funktional mit dem zweiten Ort (Arbeitsort) zu einem dritten Ort. Dieser sogenannte Third Space kann beispielsweise ein Café, ein Co-working Space oder einfach auch der Straßenraum sein, der zeitweise zu einem vergemeinschafteten Interaktionsraum wird, in dem Wissen und Informationen formell oder informell weitergegeben werden (Florida 2002: 223 ff.).

\subsection{Gesellschaftsstrukturelle raumzeitliche Veränderungen}

Der Begriff der Spätmoderne beschreibt - trotz seiner Unschärfe - die Überformung der grundsätzlichen Logiken der Moderne. Die alten fordistischen Muster, die die westlichen Industrienationen kennzeichnen, haben heute keine Allgemeingültigkeit mehr. Die Wirkmächtigkeit der Spätmoderne wird dabei zeit- und raumstrukturell mehrdimensional vermittelt (Pohl 2009: 20) und soll im Folgenden erläutert werden. Die vier Begriffe Beschleunigung, Flexibilisierung, Ausdehnung sowie Simultanität und Verdichtung sollen einerseits dazu dienen, die Analyse der gesellschaftsstrukturellen raumzeitlichen Veränderungen zu verstehen und sie andererseits als Temporalitäten fassbar zu machen. In Anlehnung an Henckel (2011: 599; vgl. auch Pohl 2009: 20 ff.; verändert und ergänzt) lassen sich die vier übergeordneten gesellschaftsstrukturellen raumzeitlichen Veränderungstendenzen in der Spätmoderne wie folgt zusammenfassen:

\section{Beschleunigung}

Die Moderne gilt als ein Zeitalter der allgemeinen Mobilmachung, die Spätmoderne hingegen steht für die Beschleunigung dieser Mobilmachung. Diese Beschleunigung findet in allen Bereichen von Wirtschaft und Gesellschaft statt. Dies deutet Rosa (2013) als eine technische Beschleunigung, die als „intentionale Steigerung der Geschwindigkeit zielgerichteter Transport-, Kommunikations- und Produktionsprozesse" (Rosa 2013: 20) zu bemerken ist. Beispielhaft seien hier die Verkürzung der Produktlebenszyklen, Beschleunigung der Verkehrsmittel oder Beschleunigung von und durch soziale Medien genannt.

\section{Flexibilisierung}

Das Aufkommen der Flexibilisierung steht für eine Erosion starrer und einheitlicher Rhythmen, die das gesellschaftliche Leben prägen. Dem Zeitalter der Industrialisierung, das mit dem Ideal einer Vollbeschäftigung einherging, steht nun eine Entgrenzung der Arbeit gegenüber, die durch Flexibilisierung der Beschäftigungsverhältnisse und die Auflösung der fordistisch-tayloristischen Normalarbeitsverhältnisse gekennzeichnet ist. Zeitliche Rhythmen werden individualisiert, von kollektiven Rhythmen abgekoppelt und von einer Reintegration der Arbeits- und Alltagswelt begleitet („Verarbeitlichung des Alltags“", Voß 1998: 482).

\section{Ausdehnung}

Zeitliche Areale, die bislang weitgehend von wirtschaftlicher Aktivität ausgenommen waren, wie etwa Wochenenden, Feiertage oder auch Abend- und Nachtzeiten, erfahren eine neue ökonomische Inwertsetzung. Der Bedeutungszuwachs von 24-Stunden- und 7-TageBeschäftigungen und der Verlust der linearen Zeitrhythmen erwirkt ein Abflachen dieser zeitlichen Übergänge. Hier sind beispielsweise Angebotsausweitungen der Freizeit- bzw. Vergnügungsökonomien in Nachtzeiten zu nennen, die unter anderem auch als Revitalisierungsmaßnahmen für vernachlässigte Quartiere eingesetzt werden (van Liempt/van Aalst/Schwanen 2015: 411).

\section{Simultanität und Verdichtung}

Mit dem Aufkommen der Möglichkeiten, mehrere Tätigkeiten gleichzeitig ausführen zu können, verschränken sich Arbeits- und Freizeiträume zunehmend. Insbesondere durch den Einsatz neuer digitaler Medien integriert die dauerhafte Erreichbarkeit durch Smartphones berufliche Aktivität stärker in andere Lebensbereiche. Dabei verschwimmen raumzeitliche Grenzen zwischen Arbeits- und Freizeitaktivität.

Diese vier übergeordneten gesellschaftsstrukturellen raumzeitlichen Veränderungstendenzen sind einerseits als Folgen und andererseits als Katalysatoren des gesellschaftlichen spätmodernen Wandels zu interpretieren. Sie sind die Folge, indem sie die gängigen bzw. überkommenen Vorstellungen von Zeit und Raum neu definieren, sie sind Katalysator, indem die ständige Suche nach Neuem und nach Abwechslung auf die veränderten raumzeitlichen Veränderungen zurückgreift und diese verändert. In einer durch Konsumismus ${ }^{2}$ und Kommodifizierung öffentlicher Räume verfassten Gesellschaft wird demnach das Ephemere begünstigt, um den

2 Bauman (2009) sieht durch den Konsumismus einen einschneidenden Wandel der Gesellschaft, der mit seinen Marktgesetzen alle Lebensbereiche durchdringt und soziale Beziehungen nachhaltig verändert. 
aktuellen gesellschaftlichen Trends immer wieder neu Rechnung zu tragen.

Diese gesellschaftlichen Zeitstrukturen haben demnach eine Flexibilisierung, Deregulierung und Beschleunigung erfahren, sodass Freizeit- und Arbeitsaktivität sich zunehmend überlagern und neue temporale Muster des gesellschaftlichen Zusammenlebens und -arbeitens entstanden sind. Die Pluralisierung und Ausdifferenzierung der Lebens- und Arbeitsstile schaffen wechselnde Synchronisationserfordernisse innerhalb neu entstandener ephemerer Arbeits- und Freizeiträume. Trotz gestiegenen Interesses fehlt es jedoch bislang ebenso an belastbaren empirischen Analysen wie an konzeptionellen Ansätzen, die temporäre lokale Ökonomien in stadtregionalen Kontexten zum Gegenstand haben. ${ }^{3}$ Vor diesem Hintergrund soll daher die im Folgenden vorgenommene Zusammenschau verschiedener Konzepte zu temporären lokalen Ökonomien einen ersten Schritt darstellen.

\section{Temporäre lokale Ökonomien und ihre beobachtbaren Phänomene}

Die Recherche zu jüngeren Arbeiten, die temporäre lokale Ökonomien aus einer raumzeitlichen Perspektive untersucht haben, ergab, dass für diese - grob zwei Perspektiven unterschieden werden können: Zum einen werden sie in Untersuchungen aus dem Bereich der Stadt- und Regionalforschung und zum anderen häufig in wissenschaftlichen Arbeiten, die der sozioökonomischen Forschung zuzurechnen sind, thematisiert. Diese fachwissenschaftlichen Perspektiven sind jedoch nicht klar voneinander zu trennen und vielfach beschäftigen sich einzelne Untersuchungen mit Aspekten beider Teilbereiche. In der Recherche stellte sich weiterhin heraus, dass insbesondere nach den 2000er-Jahren das Thema Temporalität in lokalökonomischen Kontex-

3 Die jüngste Ausnahme stellt das Schwerpunktheft „Temporäre räumliche Nähe - Akteure, Orte und Interaktionen" der Zeitschrift „Raumforschung und Raumordnung | Spatial Research and Planning"1/2020 dar(vgl. https://content.sciendo.com/view/journals/ rara/78/1/rara.78.issue-1.xml). Dort werden ausgewählte temporäre Akteurkonstellationen und ihre Bedeutung für betriebliche bzw. regionale Entwicklungsprozesse in sechs Beiträgen diskutiert. Eine der ersten Thematisierungen im deutschsprachigen Raum, in der es um Raumwirksamkeit von Zeitstrukturen und Zeitwirksamkeit von Raumstrukturen im städtischen Kontext geht, erfolgte von Henckel und Eberling (2002). ten eine größere Aufmerksamkeit erfahren hat. Arbeiten vor den 2000er-Jahren sind nur benannt, wenn sie für die weitere Entwicklung des beobachteten Fokus oder Phänomens von besonderer Bedeutung waren. Die hier aufgeführten Quellen sind jedoch nicht abschließend, sondern eher exemplarisch anzusehen (vgl. die synoptische Übersicht in Tabelle 1). Die jeweiligen Phänomene und gesellschaftsstrukturellen raumzeitlichen Veränderungen, die diese lokalen Ökonomien adressieren, sind nicht immer klar voneinander zu trennen, vielmehr überschneiden sie sich oftmals.

Folgende Kriterien lagen der Recherche fachwissenschaftlicher Arbeiten zugrunde: lokale Ökonomien (vgl. Kapitel 1), gesellschaftsstrukturelle raumzeitliche Veränderungen (vgl. Kapitel 2.2) und Erscheinungsjahr 2000 bis 2019.

\subsection{Nutzung von leerstehenden Gebäuden oder Flächen zur Revitalisierung}

Temporäre lokale Ökonomien werden oftmals im Kontext von flexiblen Zwischennutzungen diskutiert. Einen guten Überblick gibt der Sammelband von Haydn und Temel (2006), der temporäre Konzepte zur Stadtnutzung zusammenfasst. Dabei spielt Zeit „[...] eine große Rolle bei der Konstitution verschiedener Aggregatzustände leerer Räume. Ein bestimmter Zeitraum definiert den Übergang eines zeitweilig leerstehenden Hauses oder Grundstückes in eine Brache. [...] Dieser Zeitraum kann unterschiedlich groß sein, sein Endpunkt wird bestimmt durch die Wiedereingliederung der Fläche in den ordnungsgemäßen Verwertungszusammenhang. Auch andere bereits eingeführte Begriffe für leere Flächen enthalten einen Verweis auf die Zeit: Übergangsräume, Zwischenräume, Transiträume, transitorische Räume, ephemere Räume, temporäre Räume, Transformationsräume, Orte des Nicht-Mehr - Noch-Nicht, Entwicklungsräume, Vorhalteflächen“ (Dissmann 2014: 109). Diese begriffliche Vielfalt deutet an, dass diese Räume sich in einem Wandel befinden, der - je nach Ausgangspunkt - ganz unterschiedlich beschrieben werden kann. Die Räume scheinen an einen bestimmten Zeitrahmen oder -ablauf gebunden zu sein, ,... innerhalb dessen sich ein Wandel in einen anderen Zustand ereignen wird. Diese Zwischenzeit zwischen den beiden vermeintlich festen Polen 'Nicht-Mehr' und 'Noch-Nicht' ist gleichsam fluide und provisorischer Konsistenz, ein zeitlich begrenztes Stadium, bevor wieder dauerhafter gültiger Endzustand" (Dissmann 2014: 109). Dieser Ansatz des Provisori- 
Tabelle 1: Synoptische Übersicht jüngerer Arbeiten zu temporären lokalen Ökonomien (vornehmlich 2000-2019)

\begin{tabular}{|c|c|c|c|}
\hline $\begin{array}{l}\text { Gesellschaftsstrukturelle } \\
\text { raumzeitliche Veränderungen }\end{array}$ & Beobachtbare Phänomene & $\begin{array}{l}\text { Fachwissenschaftliche } \\
\text { Diskurse }\end{array}$ & Autorinnen/Autoren (Auswahl) \\
\hline Flexibilisierung & Nutzung von offenen & Zwischen- und & Overmeyer (2003); Gstach (2006); Oswalt/ \\
\hline \multirow[t]{3}{*}{ Simultanität und Verdichtung } & $\begin{array}{l}\text { Gebäuden/Flächen zur } \\
\text { Revitalisierung }\end{array}$ & Mehrfachnutzung & $\begin{array}{l}\text { Overmeyer/Misselwitz (2013); Honeck } \\
\text { (2015) }\end{array}$ \\
\hline & & $\begin{array}{l}\text { Raumunternehmer; } \\
\text { Raumpioniere }\end{array}$ & $\begin{array}{l}\text { Flögel/Gärtner (2012); Christmann/Jähnke } \\
\text { (2011); Christmann (2013); Overmeyer/ } \\
\text { Buttenberg (2014); Rink/Görbing (2019) }\end{array}$ \\
\hline & & $\begin{array}{l}\text { Zivilgesellschaftliche } \\
\text { Engagements im } \\
\text { öffentlichen Raum }\end{array}$ & $\begin{array}{l}\text { Rosol (2006); Rosol (2010); Lossau/Winter } \\
\text { (2011); Madanipour (2018) }\end{array}$ \\
\hline $\begin{array}{l}\text { Flexibilisierung } \\
\text { Ausdehnung }\end{array}$ & $\begin{array}{l}\text { Aktivitäten zu Abend- und } \\
\text { Nachtzeiten }\end{array}$ & Nachtökonomien & $\begin{array}{l}\text { Comedia Consultancy (1991); Bianchini } \\
\text { (1995); Roberts/Eldridge (2009); Shaw } \\
\text { (2014); Henckel (2015); van Liempt/van } \\
\text { Aalst/Schwanen (2015); Schwegmann } \\
\text { (2016); Weber/Henckel (2019) }\end{array}$ \\
\hline \multirow[t]{2}{*}{$\begin{array}{l}\text { Beschleunigung } \\
\text { Flexibilisierung }\end{array}$} & $\begin{array}{l}\text { Kurzzeitige Aktivitäten des } \\
\text { Handels }\end{array}$ & Mobiler Handel & $\begin{array}{l}\text { Loukaitou-Sideris/Ehrenfeucht (2009); Ha } \\
\text { (2009); Wessel (2012); Ehrenfeucht (2012); } \\
\text { Ehrenfeucht (2016); Newman/Burnett } \\
\text { (2013); Esparza/Walker/Rossman (2014); } \\
\text { Loomis (2013); Basinski (2014); Mukhija/ } \\
\text { Loukaitou-Sideris (2014); Lemon (2017); } \\
\text { Graaff/Ha (2015); Lütke (2019); Lütke } \\
\text { (2020) }\end{array}$ \\
\hline & & $\begin{array}{l}\text { Temporary Stores } \\
\text { (Pop-up-Stores; Guerilla } \\
\text { Stores) }\end{array}$ & $\begin{array}{l}\text { Hurth/Krause (2010); Surchi (2011); } \\
\text { Baumgarth/Kastner (2012); Hutter/ } \\
\text { Hoffmann (2013); Pfeufer/Suwala (2020) }\end{array}$ \\
\hline $\begin{array}{l}\text { Flexibilisierung } \\
\text { Simultanität und Verdichtung }\end{array}$ & Physische Kopräsenz & Co-working Spaces & $\begin{array}{l}\text { Lange/Wellmann (2009); Lange (2011); } \\
\text { Brinks (2012); Merkel/Oppen (2013); } \\
\text { Pelzer/Burgard (2014); Gandini (2015); } \\
\text { Merkel (2015); Butzin/Meyer (2020) }\end{array}$ \\
\hline
\end{tabular}

schen spiegelt sich sehr anschaulich im Projekt „Raum auf Zeit" wider, welches die Rolle von temporären Interventionen thematisiert, indem öffentliche Räume zum Gegenstand und Austragungsort temporärer Aktionen gemacht werden (Beeren/Berding/Kluge 2013; Berding/ Kluge 2015).

Seit Ende der 1990er-Jahre gilt der Begriff „Zwischen- und Mehrfachnutzung" vielfach als Synonym für temporäre Nutzungen leerstehender Gebäude, Gebäudeteile oder Brachflächen. Insbesondere in der Stadtund Regionalplanung gewinnen Zwischennutzungen seit dieser Zeit verstärkt Aufmerksamkeit, sodass sie im Zusammenhang mit der Planung und Durchführung von Stadtumbaumaßnahmen 2004 ins Baugesetzbuch ${ }^{4}$ aufgenommen wurden (§171a Abs. 3 Nr. 6 BauGB). ${ }^{5}$ Obwohl

4 Baugesetzbuch (BauGB) in der Fassung der Bekanntmachung vom 3. November 2017, das durch Artikel 6 des Gesetzes vom 27. März 2020 geändert worden ist.

5 Der Begriff der Zwischennutzung ist in seiner Breite nicht im Baugesetzbuch definiert. So ist aus rechtswissenschaftlicher Sicht keine Legaldefinition von Zwischennutzung besteht, gilt es in der Raumplanung als akzeptiert, dass „flexible und zeitlich befristete Nutzung von Gebäuden und Freiflächen zwischen zwei geplanten Hauptnutzungen“ (BPW 2006: 1) darunter zu verstehen sind. Hierzu zählen „von saisonalen Eventnutzungen bis zu über mehrere Jahre etablierten Standortgemeinschaften, von geduldeten Aneignungen bis zu hochpreisigen Szenelocations in Toplagen, von nachbarschaftlich gepflegten Grünflächen bis zu architektonisch durchgeplanten Großprojekten“ (BMVBS/BBR 2008: 1). Auch planungskonforme Nutzungen können nicht nur stattfinden, sondern werden

bei der wörtlichen Auslegung zwischen einer echten und einer unechten Zwischennutzung zu unterscheiden. Bei der echten Zwischennutzung ist davon auszugehen, dass sowohl die vorherige als auch die nachfolgende Nutzung feststeht und mit hinreichender Sicherheit zu erwarten ist. Eine unechte Zwischennutzung ist hingegen gegeben, wenn sich eine temporäre Nutzung ungeplant entwickelt und keine Anschlussnutzung feststeht (Austermann 2012: $146 \mathrm{ff}$.) 
erst durch die Temporalität möglich. Aus den Zwischennutzungen können auch längere Nutzungen erwachsen. Eine genaue zeitliche Grenze zwischen dauerhafter und zeitlich begrenzter Nutzung lässt sich jedoch nicht definieren (Gstach 2006: 16). Wann handelt es sich um eine dauerhafte Nutzung, wann ist es ein Provisorium? Das Herstellen eines Provisoriums lässt sich immer auch als ein spontanes Handeln im Rückgriff auf einen unmittelbaren Bezugsrahmen charakterisieren (Havemann/ Schild 2015: 44). Dabei spielt der Rückgriff auf bereits vorhandene Strukturen eine bedeutungsvolle Rolle.

Das EU-Forschungsprojekt „Urban Catalyst“ (20012003) untersuchte die Wirkung von temporären Nutzungen auf brachgefallenen Industrie- und Gewerbeflächen als Katalysator auf fünf großflächigen Brachen mit Gebäuden und Freiräumen in Europa (Amsterdam, Berlin, Helsinki, Neapel und Wien). Neben der Erkenntnis, dass Zwischennutzungen sinnvolle Handlungsansätze für die Stadtentwicklung darstellen können, wurden gerade auch große Entwicklungspotenziale für informelle Ökonomien abseits des marktwirtschaftlichen Rentabilitätsdrucks gesehen (Overmeyer 2003; Misselwitz/Oswalt/Overmeyer 2008).

Die Diskurse um die Bedeutung von lokalen Ökonomien als ökonomische Revitalisierungsmaßnahme auf Quartiersebene, wie beispielsweise beim Programm "Soziale Stadt" (vgl. Weck 2005; Prey 2014), betonen seit rund 20 Jahren die endogenen Potenziale, z. B. untergenutzte Gebäude und Flächen. Konzepte wie „Raumunternehmen“ (Flögel/Gärtner 2012; Overmeyer/ Buttenberg 2014) oder "Raumpioniere“ (Christmann/ Jähnke 2011; Christmann 2013) beziehen sich explizit auf diese Potenziale und akzentuieren die Chancen durch nutzergetragene Unternehmungen (Spars/Overmeyer 2014). Auch diese werden oft als Akteure der lokalen Ökonomien gesehen, die selbst temporäre ökonomische Arrangements generieren oder in diese eingebunden sind. „Raumunternehmen sind auf Wirtschaftlichkeit ausgerichtete Organisationen, die ihre Geschäftsidee auf Basis von unzureichend in Wert gesetzten räumlichen Ressourcen entwickeln und deren Erfolg sich an dem sozialen Nutzen für diesen Raum messen lässt" (Flögel/ Gärtner 2012: 1). Somit unterscheiden sie sich zum einen von sozialen Unternehmen, weil keine Gemeinwohlorientierung vorliegt, und zum anderen grenzen sie sich von "gewöhnlichen Unternehmen" ab, da sie einen sozialen Nutzen für den lokalen Ort erwirtschaften (Flögel/ Gärtner 2012: 5 ff.).

Hierzu gehören auch temporäre Nutzungen, wie etwa neue Formen zivilgesellschaftlichen Engagements, die als kooperative Planungsansätze zunehmend in der
Stadt- und Regionalforschung an Bedeutung gewinnen. Diese Phänomene sind im Begriff, die Art, wie Stadt gedacht und gestaltet wird, nachhaltig zu verändern. Zahlreiche realisierte Untersuchungen und Forschungsbeiträge verweisen auf neue Handlungsspielräume (Berding/Kluge 2015). In den Diskursen um temporäre Nutzungen im öffentlichen Raum, wie z. B. Gemeinschaftsgärten, in denen zivilgesellschaftliches Engagement der Bewohnerinnen und Bewohner im Fokus steht, werden randlich ökonomische Aspekte berücksichtigt: "Most of these gardens have both an economic function (food provision) and a social function (provision of social contact), irrespective of the geographical region in which they are situated" (Rosol 2010: 552).

\subsection{Aktivität zu Abend- und Nachtzeiten}

Die Nachtökonomien legen den Fokus auf die Ausdehnung wirtschaftlicher Aktivität der randlichen Tageszeiten. Van Liempt, van Aalst und Schwanen (2015: 407) werfen der Humangeographie, der Soziologie und auch den Planungswissenschaften eine bisherige „nyctalopia: night blindness" vor. Bis auf wenige Ausnahmen (Comedia Consultancy 1991; Bianchini 1995) sind erst in den letzten Jahren die urbanen Nachtökonomien verstärkt untersucht worden (Schwegmann 2016). Schwerpunkte lagen dabei in Stadtforschungen zu Clubkulturen (Lange 2007; Lange 2011) und Subkulturen (Schwanhäußer 2010; Schwanhäußer 2015) oder zu sozialen und ökonomischen Verdrängungs- und Gentrifizierungsprozessen, verbunden mit der Kritik an einer wachsenden Kommodifizierung urbaner Räume und Zeiten (Peck 2005). Seit einigen Jahren haben sich in größeren Städten (z. B. Mannheim) sogenannte Nachtbürgermeister etabliert, die Konflikte aufgrund der nächtlichen wirtschaftlichen Betätigungen im Quartier zu moderieren versuchen (Glückler/Lopez Sandoval 2019: 19).

\subsection{Kurzzeitige Aktivität des Handels}

Ein fließender Übergang, wie bei den Club- und Subkulturen, ist auch bei der mobilen Aktivität des Handels und zunehmend auch bei den Dienstleistungen zu beobachten. Der mobile Handel kann auf eine sehr lange Tradition zurückblicken. Temporäre städtische Märkte sind schon seit dem Mittelalter eine städtische wirtschaftliche Plattform, bei der insbesondere die regional verortete Nahrungsmittelproduktion wieder wichtiger zu werden scheint (Ermann 2005). Dies betrifft nicht nur die 
urbanen und stark verdichteten Räume, sondern auch ländliche Regionen, wo mobiler Handel die Grundversorgung in peripheren Lagen gewährleisten kann. Die Flexibilität, an verschiedenen Orten und nah am Kunden sein zu können, macht diese lokale Ökonomie aus. Vor dem Hintergrund einer sich stark ausdifferenzierenden Gesellschaft ist ausgehend von den USA auch in Deutschland das Phänomen der Food Trucks zu beobachten. Hierzu gehören weniger die alteingesessenen traditionellen Marktstände als vielmehr Retro-Konzepte, die in großen silberfarbenen Airstream-Wohnwagen die Konsumkultur urbaner Szeneräume bedienen (Lütke 2019; Lütke 2020). Gleichzeitig entsteht ein größeres Interesse am Straßenhandel als marginalisierte lokale Ökonomie in einer neoliberalen Stadt (Ha 2009; Graaff/ Ha 2015) oder auch an der alltäglichen Aneignung öffentlicher Räume durch informelle Straßenhändler und ihr Recht auf Stadt im globalen Süden (Etzold 2011; Etzold 2014; Mukhija/Loukaitou-Sideris 2014).

Ebenso zählen Pop-up-Stores oder Guerilla Stores zu den kurzzeitigen Aktivitäten des Handels. „Unter einem Pop-up-Store ist die einmalige, temporäre sowie gewöhnlich auf einen Standort begrenzte dreidimensionale Inszenierung einer Marke auf Handelsebene zu verstehen" (Baumgarth/Kastner 2012: 7). Im Vordergrund stehen dabei „markenauthentisches Ladendesign, produktbezogene Verknappungsinstrumente, ein erlebnisorientiertes, interaktives Unterhaltungsprogramm sowie der Einsatz von viralen Marketing-Techniken primär zur Erreichung von langfristigen, strategischen Kommunikations- und Markenzielen" (Baumgarth/Kastner 2012: 7). Die Bezeichnung Guerilla Store wird sowohl synonym zu Pop-up-Store verwendet als auch als eine Umschreibung für eine spezifische Geschäftsvariante, die verstärkt kommunikative Methoden und Instrumente des Guerilla Marketing ${ }^{6}$ einsetzt. In der Regel ist diese temporäre Art des Verkaufens ,nur' eine neue befristete Art der Verkaufsmöglichkeit von Großunternehmen, während das Hauptgeschäft weitergeführt wird (z. B. Nikes Pop-up-Stores zur Vermarktung von Sneaker im oberen Preissegment).

6 Der Begriff "Guerilla Marketing“ entstand in den 1980erJahren und beschreibt unkonventionelle kommunikative Marketinginstrumente, die oft mit einem geringen Budget zu einer hohen PR- und Medienresonanz führen. Dabei spielt das virale Marketing für ein Unternehmen eine immer wichtigere Rolle, indem z. B. in Foren, Blogs und Netzwerken ein breites (internationales) Publikum erreicht wird, „ohne im Gegenzug hohe (kommunikative) Transaktionskosten eingehen zu müssen“ (Kastner 2015: 52).

\subsection{Physische Kopräsenz}

Co-working Spaces sind flexible Schreibtischarbeitsplätze in einem Großraumbüro, die sich in den letzten Jahren rasant verbreiteten. Das Konzept basiert auf Werten, die vornehmlich in der Open Source-Bewegung zu verorten sind (Lange/Wellmann 2009: 146). Die Coworking-Idee beruht auf einer ,Kultur des Teilens' und der Kontextualisierung von Arbeitssituationen. Nicht nur der Arbeitsplatz, der Raum und die Infrastruktur sollen geteilt werden, sondern eben auch, durch die physische Kopräsenz, informelles Wissen und Ideen. Die „Coworker" kommen aus unterschiedlichen Berufsfeldern und sind vornehmlich als Selbstständige in digitalisierten Bereichen der Kreativwirtschaft tätig, die durch projektförmiges Arbeiten in dieser Branche gekennzeichnet ist. Dabei handelt es sich im Wesentlichen um immer wiederkehrende Arbeitsroutine in einem flexiblen Kontext. Durch die Betonung der personenbezogenen Netzwerke werden die Co-working Spaces zu einer neuen Form der "Marktplätze für Netzwerk-Praktiken“ (Brinks 2012: 129). Ähnlich ist dieses auch für produktionsbedingte temporäre räumliche Nähe im Einzelhandel oder Handwerk zu beobachten (Butzin/Meyer 2020). Doch ganz neu ist auch diese Idee der Kopräsenz nicht: Schon seit dem 19. Jahrhundert boten Gewerbehöfe durch die Simultanität und Verdichtung der Akteure an einem Standort vielfältige Wettbewerbsvorteile (Baumgart 2001; Gnad/Ebert/ Kunzmann 2016).

\section{Ein Beispiel lokaler temporärer Ökonomien: das Bikini Berlin}

Die vielfältigen Ausprägungen des temporären wirtschaftlichen Handelns sollen anhand eines Beispiels veranschaulicht werden, um exemplarisch die neuen temporalen Muster der ephemeren Arbeits- und Freizeiträume nachzuzeichnen.

Ein relativ junges Konzept des wirtschaftlichen Handelns auf lokaler Ebene, das temporär ausgerichtet ist, stellt ein sogenannter Pop-up-Store dar. Jüngste Beispiele sind im Gebäudekomplex „Bikini Berlin“ (vgl. Abbildung 1), dem ehemaligen "Zentrum am Zoo" in Berlins City West zu beobachten. Zu dem denkmalgeschützten Areal Bikini Berlin gehören das Bikinihaus gegenüber der Gedächtniskirche, ein größeres Hochhaus am Hardenbergplatz, das Kino „Zoo Palast”, ein 
kleineres Hochhaus mit dem „25hours Hotel“ und ein Parkhaus am Aquarium. Das Herzstück des Gebäudeensembles aus den 1950er-Jahren ist das Bikinihaus mit einer Gesamtlänge von 200 Metern. $^{7}$

Das Bikinihaus war in den 1950er-Jahren die umgangssprachliche Bezeichnung für diesen Gebäuderiegel. Einst teilte das von Säulen eingefasste Luftgeschoss im zweiten Stockwerk das Gebäude in einen oberen und einen unteren Bereich und erinnerte so an die in der Nachkriegszeit moderne zweiteilige Bademode für Frauen. Heute ist das zweite Stockwerk mit einem Fensterband zwar geschlossen, die international verständliche Bezeichnung Bikinihaus ist jedoch geblieben. Der denkmalgeschützte Gebäuderiegel von 1957 wurde als sogenannte Concept Mall 2014 wiedereröffnet. Dort befinden sich aufeinander abgestimmte Boutiquen und Gastronomiebetriebe. Eine weitere Erlebnisebene des Bikinihauses bietet die $7.000 \mathrm{~m}^{2}$ große, frei zugängliche, begrünte Dachterrasse, der „Bikini Berlin Shopping Garden", der dem New Yorker High Line Park nachempfunden ist. Um die Aufenthaltsqualität und -dauer zu erhöhen, bietet das Gebäude auf der frei zugänglichen Terrasse mit Außengastronomie einen ungewöhnlichen Blick in das Affengehege des Berliner Zoos. Das dritte bis sechste Obergeschoss des Bikinihauses beherbergt exklusive Büroflächen.

Eine Reihe von Pop-up-Stores befindet sich im Erdgeschoss des Bikinihauses, die als „a different shopping experience" (Kalandides/Millington/Parker et al. 2016: 352) in der City West vermarktet werden. Sie sind als temporär anzumietende Einheiten in der Concept Mall in Form von flexiblen Modulsystemen aus Holz angelegt (vgl. Abbildung 2). Es stehen insgesamt $20 \mathrm{Holz}-$ boxen in fünf verschiedenen Größen zur Verfügung, die drei bis maximal zwölf Monate angemietet werden können. Dort wird den Kunden ein „anderes Einkaufen“ vor allem hochwertiger Designerkleidung und -accessoires in „kuratierten Läden“ angeboten (Prossek 2015: 105). Diese provisorische Installation der kompakten Verkaufsflächen mit Bauholz suggeriert der Besucherin/ dem Besucher ein kurzweiliges und einmaliges Erlebnis und steht für die typische Wandelbarkeit von Quartieren im Zeitverlauf (Herkommer/Bormann 2017: 68). Man verzichtete auf den insgesamt $17.000 \mathrm{~m}^{2}$ auf klassische Ankermieter, um bewusst mit den sonst üblichen Shopping-Mall-Prinzipien der Innenausrichtung zu brechen, und so die Aufmerksamkeit wirksamer auf die wechselnden Konsum- und Erlebnisflächen zu lenken.

7 Vgl. https://www.bikiniberlin.de/de/about/ (24.07.2020).

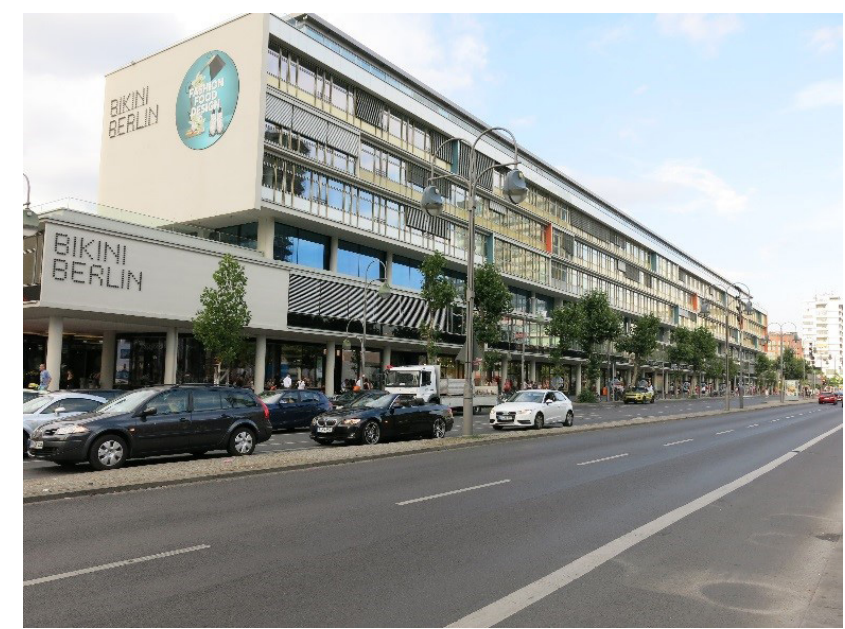

Abbildung 1: Gebäudekomplex Bikini Berlin an der Budapester Straße

Der im Innenbereich des Bikinihauses neu geschaffene Konsum- und Erlebnisraum forciert durch die ephemeren und ersetzbaren Angebote in minimalistischen Boxen die Suche nach Neuem und nach Abwechslung. Die Suche wird von einem kontinuierlichen Anbieterwechsel getragen und die Konsumabenteuer können durch die spektakulär inszenierten Räume immer wieder neu erlebt werden. Dabei greifen diese raumzeitlichen Veränderungen auf vorhandene Strukturen eines modernen und reinterpretierten Bikinihauses aus den 1950erJahren zurück und garantieren den Besucherinnen und Besuchern inszenierte und sich fortwährend erneuernde Erlebniswelten. Diese temporären Erlebniswelten spiegeln sich im Geschäftsmodell der Pop-up-Stores wider. So sprechen sie vorwiegend die Zielgruppe der Mitte 20bis Mitte 30-Jährigen an und folgen verkaufsstrategisch dem psychologischen Prinzip der Verknappung und der Exklusivität.

Mit dem Marketinginstrument der künstlichen Verknappung soll dabei der Kaufanreiz gesteigert werden, indem den Konsumierenden dadurch ein Gefühl von Exklusivität der Produkte vermittelt wird. Diese Verknappung auf der Produktebene wird begleitet von einer Verknappung auf der temporären und der räumlichen Ebene. Den Besucherinnen und Besuchern der Pop-upStores wird suggeriert, dass die Produkte nur für einen gewissen und eingeschränkten Zeitraum verfügbar sein werden, damit unterbewusst der Drang entsteht, die Produkte unmittelbar zu erstehen. Die räumliche Verknappung durch die provisorischen Stationen verstärkt diese Effekte (Gursch/Gursch 2014: 22 f.). Während der Popup-Trend sich insbesondere als Marketing- und Kommu- 


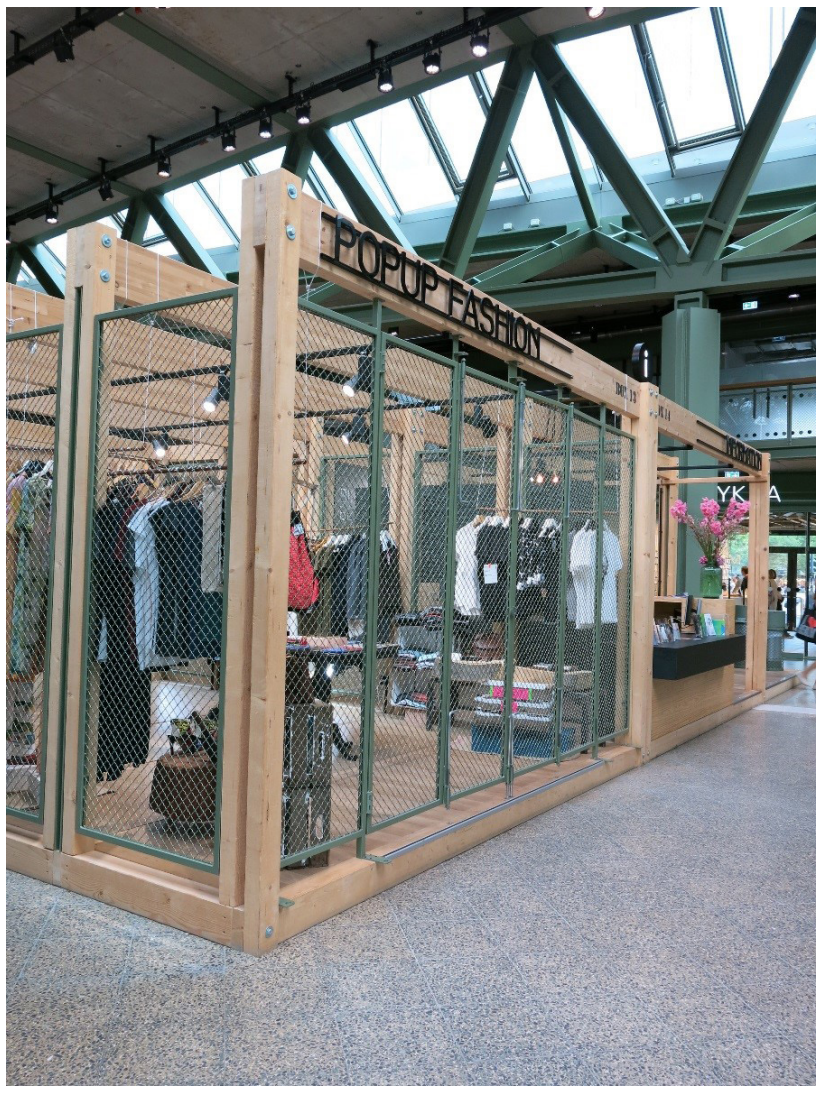

Abbildung 2: Pop-up-Stores im Innenbereich des Bikini Berlin

nikationsinstrument im Einzelhandel etabliert hat, sind die Auswirkungen auf Quartiere noch nicht abzusehen. Einerseits können die kurzfristigen Geschäftsmodelle dazu beitragen, bisher nicht oder wenig frequentierte Räume zu beleben (Harris/Nowicki 2015), andererseits besteht die Gefahr, dass es bei einer kurzfristigen unternehmerischen Aktivierung bleibt und die zunächst angezogenen Besucherströme wieder in andere Quartiere abfließen.

Im hier beschriebenen Fallbeispiel steht die symbolische Bedeutung des sich ständig erneuernden Warenangebots im Bikini Berlin auch für die Renaissance des Berliner Quartiers City West (Prossek 2015: 106). Es scheint sich genau in die von der Senatsverwaltung für Stadtentwicklung der Stadt Berlin identifizierten „Leitlinien für die City West" ${ }^{\text {"8 }}$ einzupassen, die unter anderem das Einzelhandelsprofil der City West als Kernkompetenz ausbauen sowie Kultur, Freizeit und Tourismus als Motoren der Quartiersentwicklung stärker nutzen möchten. Es bleibt jedoch abzuwarten, ob und wie diese temporären lokalen Ökonomien des Einzelhandels und

$8 \mathrm{https}: / /$ www.stadtentwicklung.berlin.de/planen/stadtplanerische konzepte/leitbild_city_west/ (24.07.2020). der Aufmerksamkeit eine nachhaltige Quartiersentwicklung anstoßen können, indem sie - z. B. auch über die Quartiersgrenzen hinweg - als touristische „Shopping Destination" in der polyzentralen Stadt Berlin wahrgenommen werden.

\section{Fazit}

Im Sinne einer spätmodernen gesellschaftlichen Entwicklung werden temporäre lokale Ökonomien und das darin eingebettete menschliche Handeln zukünftig und stärker als zuvor auf Veränderbarkeit, Bewegung und Unsicherheit ausgerichtet sein. Es entstehen so ephemere Arbeits- und Freizeiträume, die durch geringe Stabilität, zeitlich begrenzte Raumkonfigurationen und variierende Bedeutungswechsel gekennzeichnet sind. Sie sind sowohl Produkt als auch Prozess einer sich ausdifferenzierenden spätmodernen Gesellschaft. Einerseits entstehen ephemere Räume, die symbolisch aufgeladen sind und fortlaufend neu interpretiert werden. Andererseits sind es gerade diese Prozesse, die wechselseitig auf wachsende Dynamik, steigende Komplexität oder fluide Identitäten einer sich ausdifferenzierenden Gesellschaft zurückgreifen. Die wechselseitigen Beziehungen sind gekennzeichnet durch die Hybridität einer spannungsreichen Kombination von wirtschaftlichem Handeln einerseits und einer stärkeren Integration des Freizeit- und Altagslebens in wirtschaftliche Aktivität andererseits. Die raumzeitlichen Veränderungstendenzen Beschleunigung, Flexibilisierung, Ausdehnung sowie Simultanität und Verdichtung lassen dabei neue temporale Muster des gesellschaftlichen Zusammenlebens und -arbeitens entstehen.

Die in diesem Beitrag beschriebenen temporären lokalen Ökonomien und ihre konzeptionellen Diskurse zeigen die vielfältigen Entwicklungslinien im stadtregionalen Kontext auf. Das Beispiel der Pop-up-Stores im Bikinihaus in Berlin illustriert anschaulich, wie Vorstellungen von Zeit und Raum reinterpretiert werden, um aktuellen gesellschaftlichen Trends nachzukommen: die Suche nach Neuem und nach Abwechslung in einer vom Konsumismus verfassten Gesellschaft (Bauman 2009). Dadurch entstehen neue ephemere Arbeits- und Freizeiträume, die die Trennung von Arbeits- und Freizeitwelt sowie privatem und öffentlichem Raum nicht mehr zulassen.

Der verbindende Blick auf lokale Ökonomien und Temporalität kann neue Erkenntnisse sowohl konzeptionell als auch für die planerische Praxis bieten - doch gerade hier besteht weiterer Forschungsbedarf. So stellt 
sich etwa die Frage nach den unterschiedlichen Konzeptualisierungen von Temporalität und ihren theoretischen Fundierungen. Die hier angedeutete Vielfalt der wissenschaftlichen Diskurse zeigt, dass sowohl eine konzeptionelle Einbindung als auch eine theoretische Herleitung bisher wenig untersucht wurden. Ein weiteres spannendes, aber noch wenig erforschtes Feld bietet die Untersuchung temporärer lokaler Ökonomien für die nachhaltige Entwicklung von Quartieren. Den weitgehend ortsgebundenen lokalen Ökonomien fällt vor allem bei der Stabilisierung benachteiligter Quartiere mit großen immobilen Bevölkerungsanteilen eine wichtige Funktion zu. Trotz eines im Rahmen der Debatte um die integrierte Stadtteilentwicklung jüngst gestiegenen Interesses, fehlt es bislang noch an belastbaren empirischen Analysen zu temporären lokalen Ökonomien und ihren langfristigen Auswirkungen. Darüber hinaus schließt ein weiteres Forschungsfeld unmittelbar an. Aktuelle Gerechtigkeitsdiskurse, wie „Just City“ oder "Recht auf Stadt" haben gerade für die Quartiers- und Stadtentwicklung eine wichtige Bedeutung (z. B. Harvey 2008; Fainstein 2009). Nach Henckel und Kramer (2019: 5) hat die Verbindung der hochkomplexen Einzelthemen „Raum - Zeit - Gerechtigkeit“ bisher noch wenig Berücksichtigung gefunden und könnte neue Perspektiven eröffnen.

\section{Literatur}

Austermann, C. (2012): Brachflächenreaktivierung als Instrument der Stadterhaltung und nachhaltiger Innenentwicklung. Osnabrück. $=$ Planungsrecht 14 .

Basinski, S. (2014): Hot Dogs, Hipsters, and Xenophobia. Immigrant Street Food Vendors in New York. In: Social Research: An International Quarterly 81, 2, 397-408.

Bathelt, H.; Glückler, J. (2011): The relational economy. Geographies of knowing and learning. Oxford.

Bauman, Z. (2007): Leben in der flüchtigen Moderne. Frankfurt am Main.

Bauman, Z. (2009): Leben als Konsum. Hamburg.

Baumgart, S. (2001): Gewerbehöfe in der Stadt zwischen privaten Entwicklungsträgern und kommunaler Quartiersentwicklung. Dissertation an der Fakultät für Architektur und Stadtplanung der Universität Stuttgart. Stuttgart.

Baumgarth, C.; Kastner, O. L. (2012): Pop-up-Stores im Modebereich: Erfolgsfaktoren einer vergänglichen Form der Kundeninspiration. Berlin. = Working Papers of the Institute of Management Berlin at the Berlin School of Economics and Law 69.

Beeren, W.-J.; Berding, U.; Kluge, F. (2013): Raum auf Zeit. Temporäre Interventionen im öffentlichen Raum. Euskirchen.

Berding, U.; Kluge, F. (2015): Die Geschichte vom Raum auf Zeit. In: pnd online 2, 1-6.
Bianchini, F. (1995): Night Cultures, Night Economies. In: Planning Practice and Research 10, 2, 121-126. doi: 10.1080/02697459550036667

Birkhölzer, K. (2000): Formen und Reichweite lokaler Ökonomien. In: Ihmig, H. (Hrsg.): Wochenmarkt und Weltmarkt. Kommunale Alternativen zum globalen Kapital. Bielefeld, 56-88. = Impulse 8.

BMVBS - Bundesministerium für Verkehr, Bau und Stadtentwicklung; BBR - Bundesamt für Bauwesen und Raumordnung (2008): Zwischennutzungen und Nischen im Städtebau als Beitrag für eine nachhaltige Stadtentwicklung. Berlin. = Werkstatt: Praxis 57.

Bourdieu, P. (1992): Die verborgenen Mechanismen der Macht. Hamburg. = Schriften zur Politik und Kultur 1.

BPWBaumgart+Partner(2006): RecherchevonZwischennutzungen für die Überseestadt. Abschlussbericht im Auftrag der BiG Bremen. Bremen.

Brinks, V. (2012): Netzwerke(n) und Nestwärme im Coworking Space - Arbeiten zwischen Digitalisierung und Re-Lokalisierung. In: Geographische Zeitschrift 100, 3, 129-145.

Butzin, A.; Meyer, K. (2020): Urbane Produktion und temporäre räumliche Nähe in Produktionsprozessen. In: Raumforschung und Raumordnung | Spatial Research and Planning 78, 1, 5-20. doi: 10.2478/rara-2019-0061

Castells, M. (1996): The rise of the network society. Oxford.

Castells, M. (2002): Das Informationszeitalter. Teil 3: Jahrtausendwende. Opladen.

Christmann, G .B. (2013): Raumpioniere in Stadtquartieren und die kommunikative (Re-) Konstruktion von Räumen. In: Keller, R.; Knoblauch, H.; Reichertz, J. (Hrsg.): Kommunikativer Konstruktivismus. Theoretische und empirische Arbeiten zu einem neuen wissenssoziologischen Ansatz. Wiesbaden, 153184. doi: 10.1007/978-3-531-19797-5_7

Christmann, G. B.; Jähnke, P. (2011): Soziale Probleme und innovative Ansätze in der Quartiersentwicklung. In: Jähnke, P.; Christmann, G. B.; Balgar, K. (Hrsg.): Social Entrepreneurship. Perspektiven für die Raumentwicklung. Wiesbaden, 211-234. doi: 10.1007/978-3-531-92819-7_17

Comedia Consultancy (1991): Out of hours: a study of economic, social and cultural life in twelve town centres in the UK. London.

Dissmann, C. (2014): Die Gestaltung der Leere. Zum Umgang mit einer neuen städtischen Wirklichkeit. Bielefeld.

Ehrenfeucht, R. (2012): Precursors to Planning. Regulating the Streets of Los Angeles, California, 1880-1920. In: Journal of Planning History 11, 2, 107-123. doi: 10.1177/1538513211428275

Ehrenfeucht, R. (2016): Designing Fair and Effective Street Vending Policy: It's Time for a New Approach. In: Cityscape 18, 1, 11-26.

Elbe, M.; Peters, S. (2016): Die temporäre Organisation. Grundlagen der Kooperation, Gestaltung und Beratung. Berlin.

Ermann, U. (2005): Regionalprodukte. Vernetzungen und Grenzziehungen beider Regionalisierung von Nahrungsmitteln. Stuttgart. = Sozialgeographische Bibliothek 3.

Esparza, N.; Walker, E. T.; Rossman, G. (2014): Trade Associations and the Legitimation of Entrepreneurial Movements: Collective Action in the Emerging Gourmet Food Truck Industry. In: Nonprofit and Voluntary Sector Quarterly 43, 2, 143-162. doi: 10.1177/0899764013512723

Etzold, B. (2011): Die umkämpfte Stadt. Die alltägliche Aneignung öffentlicher Räume durch Straßenhändler in Dhaka 
(Bangladesch). In: Holm, A.; Gebhardt, D. (Hrsg.): Initiativen für ein Recht auf Stadt. Theorie und Praxis städtischer Aneignungen. Hamburg, 187-220.

Etzold, B. (2014): Raumaneignungen, Regeln und Profite in Dhakas Feld des Straßenhandels - Sozialgeographische Erklärungsversuche auf Grundlage von Bourdieus Theorie der Praxis. In: Geographica Helvetica 69, 1, 37-48. doi: 10.5194/ gh-69-37-2014

Fainstein, S. (2009): Planning and the Just City. In: Marcuse, P.; Connolly, J.; Novy, J.; Olivo, I.; Potter, C.; Steil, J. (Hrsg.): Searching for the just city: Debates in urban theory and practice. London, 19-40.

Flögel, F.; Gärtner, S. (2012): Raumunternehmen und die Aktivierung von Nachbarschaften. Gelsenkirchen.

Florida, R. (2002): The rise of the creative class. And how it's transforming work, leisure, community and everyday life. New York.

Franck, G. (1998): Ökonomie der Aufmerksamkeit. Ein Entwurf. München.

Gandini, A. (2015): The rise of coworking spaces. A literature review. In: Ephemera. Theory and Politics in Organization 15, 1, 193-205.

Giddens, A. (1992): Die Konstitution der Gesellschaft. Grundzüge einer Theorie der Strukturierung. Frankfurt am Main.

Glückler, J.; Lopez Sandoval, C. (2019): Die Clubszene in Heidelberg. Heidelberg.

Gnad, F.; Ebert, R.; Kunzmann, K. R. (2016): Kultur- und Kreativwirtschaft in Stadt und Region. Branchen - Orte Netze. Stuttgart.

Graaff, K.; Ha, N. (2015): Introduction. Street Vending in the Neoliberal City: A Global Perspective on the Practices and Policies of a Marginalized Economy. In: Graaff, K.; Ha, N. (Hrsg.): Street Vending in the Neoliberal City. A Global Perspective on the Practices and Policies of a Marginalized Economy. New York, 1-15.

Gstach, D. (2006): Freiräume auf Zeit. Zwischennutzung von urbanen Brachen als Gegenstand der kommunalen Freiraumentwicklung. Dissertation am Fachbereich 06 Architektur Stadtplanung Landschaftsplanung der Universität Kassel. Kassel.

Güleş, O.; Schultheis, K. (2019): Förderkonzept von BIWAQ im Handlungsfeld lokale Ökonomie. In: Niermann, O.; Schnur, O.; Drilling, M. (Hrsg.): Ökonomie im Quartier. Von der sozialräumlichen Intervention zur Postwachstumsgesellschaft. Wiesbaden, 9-27. doi: 10.1007/978-3-658-23446-1_2

Gursch, F.; Gursch, G. (2014): Die Erfolgsfaktoren von Pop-upStores. In: Ternès, A.; Towers, I. (Hrsg.): Internationale Trends in der Markenkommunikation. Was Globalisierung, neue Medien und Nachhaltigkeit erfordern. Wiesbaden, 15-31. doi: 10.1007/978-3-658-01517-6_2

Ha, N. K. (2009): Informeller Straßenhandel in Berlin. Urbane Raumproduktion zwischen Störung und Attraktion. Berlin.

Hägerstrand, T. (1970): What about people in regional science? In: Papers in Regional Science 24, 1, 7-24. doi: 10.1111/j.14355597.1970.tb01464.x

Hägerstrand, T. (1975): Space, time and human conditions. In: Karlqvist, A.; Lundqvist, L.; Snickars, F. (Hrsg.): Dynamic allocation of urban space. Stockholm conference. Farnborough, 3-12.
Harris, E.; Nowicki, M. (2015): Cult of the temporary: is the pop-up phenomenon good for cities? https://www.theguardian.com/ cities/2015/jul/20/cult-temporary-pop-up-phenomenon-cities (24.07.2020).

Harvey, D. (1990): The Condition of Postmodernity: An Enquiry into the Origins of Cultural Change. Oxford.

Harvey, D. (2008): The right to the city. In: New Left Review 53, 23-40.

Havemann, A.; Schild, M. (2015): „Das machen wir erst einmal so!“ Provisorien und die Fähigkeit zu Handeln. In: Raumplanung 178, 2, 43-47.

Haydn, F.; Temel, R. (Hrsg.) (2006): Temporäre Räume. Konzepte zur Stadtnutzung. Basel.

Henckel, D.; Eberling, M. (Hrsg.) (2002): Raumzeitpolitik. Opladen. Henckel, D. (2011): Beschleunigung - Effizienzsteigerung oder Zeitverlust? In: Informationen zur Raumentwicklung 10, 599607.

Henckel, D. (2015): Die Nacht, das Nachtleben und die Stadt. Die Nacht gewinnt an Bedeutung. Interview mit Prof. Dr. Dietrich Henckel. In: BBSR - Bundesinstitut für Bau-, Stadt- und Raumforschung (Hrsg.): Stadt:pilot spezial: Die Stadt und das Nachtleben. Bonn, 6-9.

Henckel, D.; Kramer, C. (2019): Zeitgerechte Stadt - eine Einführung. In: Henckel, D.; Kramer, C. (Hrsg.): Zeitgerechte Stadt - Konzepte und Perspektiven für die Planungspraxis. Hannover, 5-22. = Forschungsberichte der ARL 9.

Henn, S.; Behling, M. (2020): Lokale Ökonomie - Begriff, Merkmale und konzeptionelle Abgrenzung. In: Henn, S.; Behling, M.; Schäfer, S. (Hrsg.): Lokale Ökonomie. Konzepte, Quartierskontexte und Interventionen. Berlin, 3-24. doi: 10.1007/978-3-662-57780-6_1

Herkommer, B.; Bormann, D. (2017): Placemaking. Die Kunst, gute Orte auszubilden. In: Besecke, A.; Meier, J.; Pätzold, R.; Thomaier, S. (Hrsg.): Stadtökonomie - Blickwinkel und Perspektiven. Ein Gemischtwarenladen. Berlin, 62-69.

Honeck, T. (2015): Zwischennutzung als soziale Innovation. Von alternativen Lebensentwürfen zu Verfahren der räumlichen Planung. In: Informationen zur Raumentwicklung 3, 219-231.

Hurth, J.; Krause, M. (2010): Ortswechsel: Pop-Up-Stores als innovativer Betriebstyp. In: Transfer, Werbeforschung und Praxis 56, 1, 33-40.

Hutter, K.; Hoffmann, S. (2013): Professionelles Guerilla-Marketing. Grundlagen - Instrumente - Controlling. Wiesbaden. doi: 10.1007/978-3-658-02268-6

Ibert, O.; Thiel, J. (2009): Situierte Analyse, dynamische Räumlichkeiten. In: Zeitschrift für Wirtschaftsgeographie 53, 1-2, 209-223. doi: 10.1515/zfw.2009.0015

Jameson, F. (2003): The end of temporality. In: Critical Inquiry 29, 4, 695-718.

Kaiser, R.; Nikiforova, E. (2008): The performativity of scale. The social construction of scale effects in Narva, Estonia. In: Environment and Planning D: Society and Space 26, 3, 537562. doi: $10.1068 / \mathrm{d} 3307$

Kalandides, A.; Millington, S.; Parker, C.; Quin, S. (2016): Shopping districts and centres, markets, neighbourhoods, public squares, and urban gardens: Reflecting upon place management practice in Berlin. In: Journal of Place Management and Development 9, 3, 351-359. doi: 10.1108/JPMD-09-2016-0059 
Kastner, O. L. (2015): Erfolgsfaktoren von Pop-up Stores. Fallstudiengestützte Evaluation am Beispiel der Bekleidungsindustrie. Wiesbaden. doi: 10.1007/978-3-65808945-0

Klamt, M. (2012): Öffentliche Räume. In: Eckardt, F. (Hrsg.): Handbuch Stadtsoziologie. Wiesbaden, 775-804. doi: 10.1007/978-3-531-94112-7_34

Koch, J.; Sydow, J. (2013): Vorwort. In: Koch, J.; Sydow, J. (Hrsg.): Organisation von Temporalität und Temporärem. Wiesbaden, VII-IX. = Managementforschung 23. doi: 10.1007/978-3-65802998-2

Kramer, C. (2005): Zeit für Mobilität. Räumliche Disparitäten der individuellen Zeitverwendung für Mobilität in Deutschland. Stuttgart. $=$ Erdkundliches Wissen 138.

Kymäläinen, P.; Nordström, P. (2010): Temporary geographies of the city. The experienced spaces of asylum seekers in the City of Turku, Finland. In: Fennia - International Journal of Geography 188, 1, 76-90.

Läpple, D.; Mückenberger, U.; Oßenbrügge, J. (Hrsg.) (2010): Zeiten und Räume der Stadt. Theorie und Praxis. Leverkusen.

Lange, B. (2007): Die Räume der Kreativszenen. Culturepreneurs und ihre Orte in Berlin. Bielefeld.

Lange, B. (2011): Neue Organisationsformen in der Kultur- und Kreativwirtschaft. In: BMVBS - Bundesministerium für Verkehr, Bau und Stadtentwicklung (Hrsg.): Kultur- und Kreativwirtschaft in Stadt und Region. Voraussetzungen, Handlungsstrategien und Governance. Berlin, 52-62.

Lange, B.; Wellmann, I. (2009): „Neue Orte für neues Arbeiten“ 1. Co-working Spaces. In: Lange, B.; Kalandides, A.; Stöber, B.; Wellmann, I. (Hrsg.): Governance der Kreativwirtschaft. Diagnosen und Handlungsoptionen. Bielefeld, 145-152.

Lemon, R. (2017): The Spatial Practices of Food Trucks. In: Agyeman, J.; Matthews, C.; Sobel, H. (Hrsg.): Food trucks, cultural identity, and social justice: From loncheras to lobsta love. Cambridge, 169-188.

Lenntorp, B. (1999): Time-geography - at the end of its beginning. In: GeoJournal 48, 3, 155-158. doi: 10.1023/A:1007067322523

Loomis, J. (2013): Moveable Feasts: Locating Food Trucks in the Cultural Economy. Master Thesis am College of Arts and Sciences der University of Kentucky. Lexington, Kentucky.

Lossau, J.; Winter, K. (2011): The social construction of city nature: exploring temporary uses of open green space in Berlin. In: Endlicher, W.; Hostert, P.; Kowarik, I.; Kulke, E.; Lossau, J.; Marzluff, J.; van der Meer, E.; Mieg, H.; Nützmann, G.; Schulz, M.; Wessolek, G. (Hrsg.): Perspectives in urban ecology. Studies of ecosystems and interactions between humans and nature in the metropolis of Berlin. Berlin, 333-345. doi: 10.1007/978-3-642-17731-6_12

Loukaitou-Sideris, A.; Ehrenfeucht, R. (2009): Sidewalks. Conflict and negotiation over public space. Cambridge.

Lütke, P. (2019): Die Praxis temporärer Ökonomien im Quartier Hubs, Flows und Persistencies des "mobile food vending“ in den USA. In: Niermann, O.; Schnur, O.; Drilling, M. (Hrsg.): Ökonomie im Quartier. Von der sozialräumlichen Intervention zur Postwachstumsgesellschaft. Wiesbaden, 217-235. doi: 10.1007/978-3-658-23446-1_12

Lütke, P. (2020): Temporäre Ökonomien in der postmodernen Stadt. In: Gebhardt, H.; Glaser, R.; Radtke, U.; Reuber, P.; Vött, A. (Hrsg.): Geographie - Physische Geographie und Humangeographie. Berlin, 865.
Madanipour, A. (2018): Temporary use of space. Urban processes between flexibility, opportunity and precarity. In: Urban Studies 55, 5, 1093-1110. doi: 10.1177/0042098017705546

Massey, D. (1984): Spatial Divisions of Labour. Social Structures and the Geography of Production. London.

Massey, D. (2005): For space. Los Angeles.

May, J.; Thrift, N. J. (Hrsg.) (2001): Timespace. Geographies of temporality. London. $=$ Critical geographies 13 .

Merkel, J. (2015): Coworking in the City. In: Ephemera. Theory and Politics in Organization 15, 1, 121-139.

Merkel, J.; Oppen, M. (2013): Coworking Spaces. Die (Re-) Organisation kreativer Arbeit. Berlin. = WZBrief Arbeit 16.

Misselwitz, P.; Oswalt, P.; Overmeyer, K. (2008): Stadtentwicklung ohne Städtebau - Planerischer Alptraum oder gelobtes Land? In: Schmitt, G.; Selle, K. (Hrsg.): Bestand? Perspektiven für das Wohnen in der Stadt. Dortmund, 628-637.

Mukhija, V.; Loukaitou-Sideris, A. (2014): The Informal American City. Beyond Taco Trucks and Day Labor. Cambridge.

Newman, L. L.; Burnett, K. (2013): Street food and vibrant urban spaces. Lessons from Portland, Oregon. In: Local Environment 18, 2, 233-248. doi: 10.1080/13549839.2012.729572

Oinas, P.; Malecki, E. J. (2002): The Evolution of Technologies in Time and Space. From National and Regional to Spatial Innovation Systems. In: International Regional Science Review 25, 1, 102-131. doi: 10.1177/016001702762039402

Oswalt, P.; Overmeyer, K.; Misselwitz, P. (Hrsg.) (2013): Urban Catalyst. Mit Zwischennutzungen Stadt entwickeln. Berlin.

Overmeyer, K. (2003): Urbane Sukzession. In: Anthos 42, 2, 41-45.

Overmeyer, K.; Buttenberg, L. (2014): Raumunternehmen zwischen informeller Aktivierung und formeller Planung. In: Informationen zur Raumentwicklung 2, 135-142.

Padgett, J.F.; Powell, W.W. (2012): The Emergence of Organizations and Markets. Princeton.

Peck, J. (2005): Struggling with the Creative Class. In: International Journal of Urban and Regional Research 29, 4, 740-770. doi: 10.1111/j.1468-2427.2005.00620.x

Pelzer, C.; Burgard, N. (2014): Co-Economy: Wertschöpfung im digitalen Zeitalter. Netzwerke und agile Organisationsstrukturen erfolgreich nutzen. Wiesbaden. doi: 10.1007/978-3-65800955-7

Pfeufer, N.; Suwala, L. (2020): Inwertsetzung von temporären Räumlichkeiten. Standortstrategien von Pop-up-Restaurants in Berlin. In: Raumforschung und Raumordnung | Spatial Research and Planning 78, 1, 71-87. doi: 10.2478/rara-20190053

Pohl, T. (2009): Entgrenzte Stadt. Räumliche Fragmentierung und zeitliche Flexibilisierung in der Spätmoderne. Bielefeld.

Prey, G. (2014): Von Stadtmarketing, BIDs und ISGs: Neue Governance-Formen in der Quartiers-Ökonomie. In: Schnur, O. (Hrsg.): Quartiersforschung. Zwischen Theorie und Praxis. Wiesbaden, 293-314. doi: 10.1007/978-3-531-19963-4_14

Prossek, A. (2015): Berlins City-West verändert ihr Gesicht. In: Standort - Zeitschrift für Angewandte Geographie 39, 2-3, 102-107. doi: 10.1007/s00548-015-0382-0

Rilling, R. (2014): Transformation als Futuring. In: Brie, M. (Hrsg.): Futuring. Perspektiven der Transformation im Kapitalismus über inn hinaus. Münster, 12-48.

Rink, D.; Görbing, M. (2019): Zwischennutzungen in unterschiedlichen urbanen Kontexten. Die Beispiele Leipzig und Dessau-Roßlau. In: Raumforschung und Raumordnung 
| Spatial Research and Planning 77, 6, 601-615. doi: 10.2478/ rara-2019-0041

Roberts, M.; Eldridge, A. (2009): Planning the night-time city. London.

Rosa, H. (2013): Beschleunigung und Entfremdung. Entwurf einer kritischen Theorie spätmoderner Zeitlichkeit. Berlin.

Rosa, H. (2017): Beschleunigung: Die Veränderung der Zeitstrukturen in der Moderne. Frankfurt am Main.

Rosol, M. (2006): Gemeinschaftsgärten in Berlin. Eine qualitative Untersuchung zu Potenzialen und Risiken bürgerschaftlichen Engagements im Grünflächenbereich vor dem Hintergrund des Wandels von Staat und Planung. Berlin.

Rosol, M. (2010): Public participation in post-Fordist urban green space governance. The case of community gardens in Berlin. In: International Journal of Urban and Regional Research 34, 3, 548-563. doi: 10.1111/j.1468-2427.2010.00968.x

Rummel, M. (2011): Wer sind Social Entrepreneurs in Deutschland? Soziologischer Versuch einer Profilschärfung. Wiesbaden. doi: 10.1007/978-3-531-94152-3

Sassen, S. (2002): Towards post-national and denationalized citizenship. In: Isin, E. F.; Turner, B. S. (Hrsg.): Handbook of Citizenship Studies. London, 277-292.

Scheiner, J. (1998): Aktionsraumforschung auf phänomenologischer und handlungstheoretischer Grundlage. In: Geographische Zeitschrift 86, 1, 50-66.

Schwanhäußer, A. (2010): Kosmonauten des Underground. Ethnografie einer Berliner Szene. Frankfurt am Main. = Interdisziplinäre Stadtforschung 7.

Schwanhäußer, A. (2015): Herumhängen. Stadtforschung aus der Subkultur. In: Zeitschrift für Volkskunde 111, 1, 76-93.

Schwegmann, R. (2016): Nacht-Orte. Eine kulturelle Geographie der Ökonomie. Bielefeld. = Sozial- und Kulturgeographie 12.

Shaw, R. (2014): Beyond night-time economy. Affective atmospheres of the urban night. In: Geoforum 51, 87-95. doi: 10.1016/j.geoforum.2013.10.005

Spars, G.; Overmeyer, K. (2014): Raumunternehmen als treibende Kraft der Quartiersentwicklung. In: Schnur, O.; Drilling, M.; Niermann, O. (Hrsg.): Zwischen Lebenswelt und Renditeobjekt. Quartiere als Wohn- und Investitionsorte. Wiesbaden, 159-173. doi: 10.1007/978-3-658-06161-6_9

Surchi, M. (2011): The temporary store. A new marketing tool for fashion brands. In: Journal of Fashion Marketing and Management 15, 2, 257-270. doi: 10.1108/13612021111132672

Törnqvist, G. (2004): Creativity in Time and Space. In: Geografiska Annaler 86, 4, 227-243. doi: 10.1111/j.0435-3684.2004.00165.x

van Liempt, I.; van Aalst, I.; Schwanen, T. (2015): Introduction: Geographies of the urban night. In: Urban Studies 52, 3, 407421. doi: $10.1177 / 0042098014552933$

Voß, G. G. (1998): Die Entgrenzung von Arbeit und Arbeitskraft. Eine subjektorientierte Interpretation des Wandels der Arbeit. In: Mitteilungen aus der Arbeitsmarkt- und Berufsforschung 31, 3, 473-487.

Weber, C.; Henckel, D. (2019): Nacht und Gerechtigkeit - die Stadtnacht als spezifischer Zeit-Raum. In: Henckel, D.; Kramer, C. (Hrsg.): Zeitgerechte Stadt - Konzepte und Perspektiven für die Planungspraxis. Hannover, 109-139. = Forschungsberichte der ARL 9.
Weck, S. (2005): Quartiersökonomie im Spiegel unterschiedlicher Diskurse. Standpunkte und theoretische Grundlagen zur Revitalisierung erneuerungsbedürftiger Stadtteile. Dortmund. = Dortmunder Beiträge zur Raumplanung 124.

Wessel, G. (2012): From Place to NonPlace. A Case Study of Social Media and Contemporary Food Trucks. In: Journal of Urban Design 17, 4, 511-531. doi: 10.1080/13574809.2012.706362

Wood, G. (2003): Die Wahrnehmung städtischen Wandels in der Postmoderne. Untersucht am Beispiel der Stadt Oberhausen. Opladen. $=$ Stadtforschung aktuell 88. 